\title{
ANOMALOUS PRESSURE-INDUCED CHANGES IN MAGNETISM OF FERRIMAGNETIC Ni-Mn-Sn STRUCTURES
}

\author{
${ }^{1}$ Martin FRIÁK, ${ }^{1,2}$ Martina MAZALOVÁ, ${ }^{1}$ Ivana MIHÁLIKOVÁ, ${ }^{2,1}$ Mojmír ŠOB \\ ${ }^{1}$ Institute of Physics of Materials, v.v.i., Czech Academy of Sciences, Brno, Czech Republic, EU, \\ friak@ipm.cz, 394206@mail.muni.cz,.mihalikova@ipm.cz \\ ${ }^{2}$ Department of Chemistry, Faculty of Science, Masaryk University, Brno, Czech Republic, EU \\ mojmir@ipm.cz
}

https://doi.org/10.37904/nanocon.2021.4306

\begin{abstract}
We have performed a quantum-mechanical study of two ferrimagnetic $\mathrm{Ni}_{2} \mathrm{MnSn}$ structures, a stoichiometric one with $\mathrm{Mn}-\mathrm{Ni}$ swaps and an off-stoichiometric $\left(\mathrm{Ni}_{7} \mathrm{Mn}_{8} \mathrm{Sn}_{1}\right)$ alloy. We used 16-atom supercells with the atomic positions related to those in the full Heusler structure, i.e. austenite phase (unless distorted by internal distribution of point defects as in the case of $\mathrm{Ni}_{2} \mathrm{MnSn}$ with swaps). We have determined thermodynamic, magnetic and structural properties of both pressure-free states as well as those corresponding to hydrostatic pressures of a few GPa. The atomic and magnetic configurations of the studied states are found to exhibit anomalous pressure-induced changes in the total magnetic moment. In particular, the total magnetic moments per 16-atom supercells increase with increasing hydrostatic pressure. Despite this peculiar trend in the total magnetic moment, the magnitudes of local magnetic moments of $\mathrm{Mn}$ atoms, that are decisive for the value of the total magnetic moment, decrease with increasing hydrostatic pressure (as is common in majority of magnetic systems). The identified phenomena may be related to an interplay of a few contributing mechanisms. First, the magnetic moments of $\mathrm{Mn}$ atoms, that are either parallel or antiparallel to the orientation of the total magnetic moment of the supercells, nearly compensate each other due to the ferrimagnetic nature of the studied magnetic states. Second, the swapped and off-stoichiometric atoms lead to different local atomic environments of $\mathrm{Mn}$ atoms and, consequently, to different local magnetic moments of these atoms as well as their different response to hydrostatic pressures. Importantly, the local magnetic moments of $\mathrm{Mn}$ atoms, that are antiparallel to the orientation of the total magnetic moment, are more sensitive to the applied pressures. Regarding thermodynamics, the studied states are excited ones.
\end{abstract}

Keywords: Ni-Mn-Sn, magnetism, pressure, ferrimagnetic, austenite, quantum-mechanical, defects

\section{INTRODUCTION}

One of the most intensively studied materials is ternary $\mathrm{X}_{2} \mathrm{YZ}$ materials with Heusler-type crystal lattice [1]. The Heusler alloys include numerous combinations of different chemical elements and some of them possess an extraordinary magneto-structural transitions that are often accompanied by significant changes of their properties [2]. In particular, the off-stoichiometric $\mathrm{Ni}_{2} \mathrm{MnX}(\mathrm{X}=\mathrm{Ga}, \mathrm{In}, \mathrm{Sn})$ compounds exhibit unusual magnetic behavior stemming from competing antiferromagnetic and ferromagnetic interactions between magnetic sublattices with $\mathrm{Mn}$ atoms [2-5]. The stoichiometric austenite $\mathrm{Ni}_{2} \mathrm{Mn}$-based alloys possess a long range ferromagnetic arrangement of moments below the Curie temperature, see, e.g. Ref. [7]. In contrast, the Ni$\mathrm{Mn}-\mathrm{X}$ compounds undergo several magnetic and magneto-structural transition [8-10] and some of these interesting phenomena are not fully understood yet. Our study was motivated by the current interest in pressure-induced changes in Ni-Mn-Sn structures [7] when an unexpected complexity is often found depending on the type and distribution of internal defects as well as their magnetic state, see, e.g. [11]. 
Before we proceed to describing two ferrimagnetic Ni-Mn-Sn structures, that we have studied, it is worth making a few comments on the structure and properties of the stoichiometric austenitic $\mathrm{Ni}_{2} \mathrm{MnSn}_{\mathrm{S}}$ without any defects. Its atomic distribution is that of the full Heusler structure, see Figure 1. The lowest energy configuration exhibits local magnetic moments of atoms as listed in Figure 1(a); further details can be found in our recent paper [11]. The magnetic state is, in principle, ferrimagnetic due to the orientation of local magnetic moment of $\mathrm{Sn}$ atoms that is antiparallel to those of $\mathrm{Mn}$ and $\mathrm{Ni}$ atoms. But the local magnetic moments of $\mathrm{Sn}$ atoms are equal only to $0.05 \mu$ B, i.e. nearly an order of magnitude smaller than the magnitudes of local magnetic moments of $\mathrm{Ni}$ atoms and nearly two orders of magnitude smaller than those of $\mathrm{Mn}$ atoms. In fact, the magnitudes and orientations of $\mathrm{Mn}$ atoms are often decisive for the total magnetic moment of $\mathrm{Ni}-\mathrm{Mn}-\mathrm{Sn}$ systems. Therefore, the state shown in Figure 1(a) is often called ferromagnetic reflecting the ferromagnetic orientation of local magnetic moments of $\mathrm{Mn}$ atoms, as well as of $\mathrm{Ni}$ atoms, with respect to the orientation of the total magnetic moment. Our previous study [11] of various magnetic and structural variants of stoichiometric $\mathrm{Ni}_{2} \mathrm{MnSn}$ with different types of swapped atoms revealed that the response to the hydrostatic pressures is highly sensitive to both atomic configuration and magnetic state.

\section{COMPUTATIONAL METHODOLOGY}

As a continuation of our previous research [11], we have computed pressure-induced changes in the case of two ferrimagnetic Ni-Mn-Sn states. One was the stoichiometric $\mathrm{Ni}_{2} \mathrm{MnSn}$ case with two Ni-Mn swaps and the second quite off-stoichiometric $\mathrm{Mn}$-rich and $\mathrm{Sn}$-poor alloy with the stoichiometry $\mathrm{Ni}_{7} \mathrm{Mn}_{8} \mathrm{Sn}_{1}$. Both were modeled using specific variants of the 16-atom supercell shown in Figure 1(b). The supercell of the former state has a tetragonal symmetry while the latter one possesses a cubic one (details will be discussed below).

The Vienna Ab initio Simulation package (VASP) $[12,13]$ implementing the density functional theory (DFT) $[14,15]$ was used to perform our ab initio calculations. We have employed projector augmented wave (PAW) $[16,17]$ pseudopotentials (Ni_pv, Mn_pv and Sn_d versions from the potpaw_PBE.52 VASP database) and the generalized gradient approximation (GGA) as parametrized by Perdew, Burke and Ernzerhof (PBE'96), see Ref. [18]. In the case of 16-atom supercells, the plane-wave energy cut-off was set to $700 \mathrm{eV}$ and the $8 \times 8 \times 8$ k-point mesh with its origin shifted to $(1 / 2,1 / 2,1 / 2)$ was used. We applied the Methfessel-Paxton order 1 smearing with the smearing parameter set to 0.23 . Supercells modeling the impact of hydrostatic pressures were partly relaxed, i.e., their total energy was minimized with respect to the atomic positions and the cell shape for a given volume corresponding to a certain applied hydrostatic pressure.

(a)

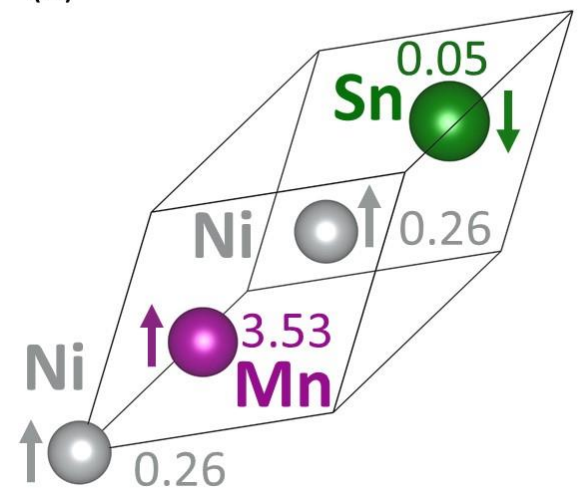

(b)

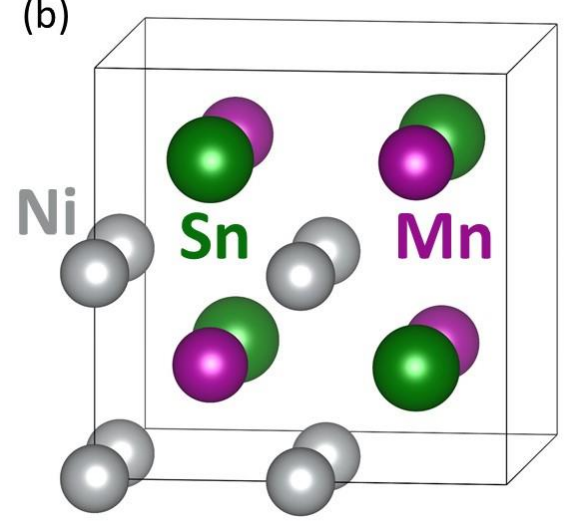

Figure 1 Schematic visualizations of (a) 4-atom rhombohedral primitive unit cell and (b) 16-atom cubeshaped conventional supercell of Heusler-structure (i.e. austenitic) $\mathrm{Ni}_{2} \mathrm{MnSn}$ without any defects. The latter supercell was used for our modeling of states with a pair of Mn-Ni swaps and an off-stoichiometric $\mathrm{Ni}_{7} \mathrm{Mn}_{8} \mathrm{Sn}_{1}$ alloy. Sub-figure (a) contains also computed values of local magnetic moments of atoms in Bohr magnetons and their orientation indicated by arrows, for details see Ref. [11]. 


\section{RESULTS}

Figure 2(a) visualizes the atomic configuration of the stoichiometric allotrope of $\mathrm{Ni}_{2} \mathrm{MnSn}$ with two $\mathrm{Ni}-\mathrm{Mn}$ swaps per 16-atom supercell. We focused on a ferrimagnetic configuration when the swapped $\mathrm{Mn}$ atoms on the $\mathrm{Ni}$ sublattice have their local magnetic moments antiparallel to local magnetic moments of the two remaining $\mathrm{Mn}$ atoms on the Mn sublattice as presented in Figure 2(b). When minimizing the energy for this configuration, i.e. the state without any hydrostatic pressure, the swapped $\mathrm{Mn}$ atoms on the Ni sublattice turned out to have the magnitude of the local magnetic moments $(2.78 \mu \mathrm{B})$ lower than the Mn atoms on the Mn sublattice (3.49 $\mu \mathrm{B})$ by 0.71 Bohr magneton, see Figure 2(b). Contrary to this trend, the $\mathrm{Ni}$ atoms on the Mn sublattice have the magnitude of their local magnetic moments $(0.25 \mu \mathrm{B})$ higher than those of $\mathrm{Ni}$ atoms on the Ni sublattice $(0.9-0.14 \mu \mathrm{B})$. The orientation of $\mathrm{Ni}$ atoms on the $\mathrm{Mn}$ sublattice is antiparallel to that of local magnetic moments of $\mathrm{Ni}$ atoms on the Ni sublattice (and this orientation of the total magnetic moment is also that of the whole 16atom supercell). The magnitude of the total magnetic moment is only $1.77 \mu \mathrm{B}$ per 16 -atom supercell due to a nearly complete compensation of parallel and antiparallel local magnetic moments of atoms. The pressuredependence of the total magnetic moment of the whole supercell, see Figure 2(c), has an anomalous trend when it increases for increasing pressure, i.e. decreasing volume.

(a)

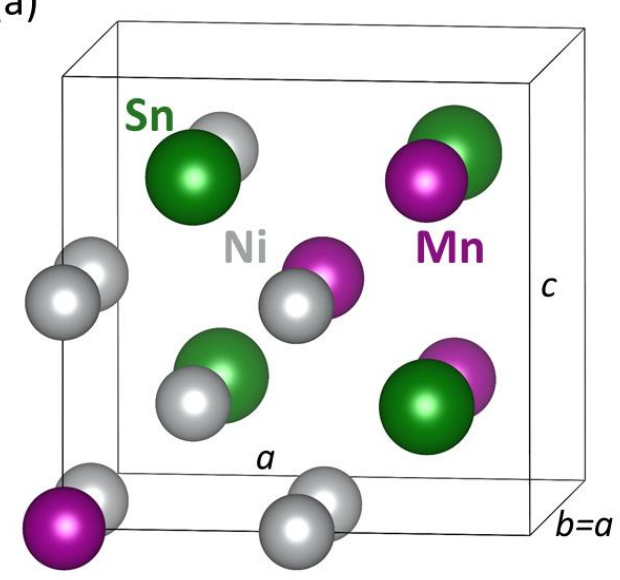

(c)

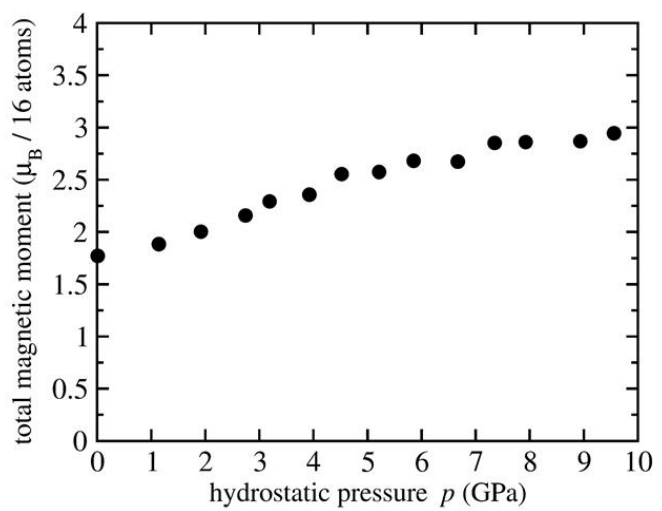

(b)

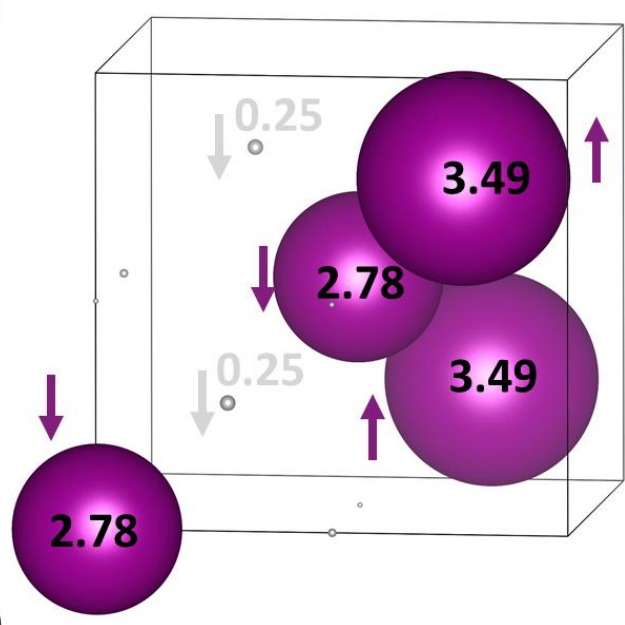

(d)

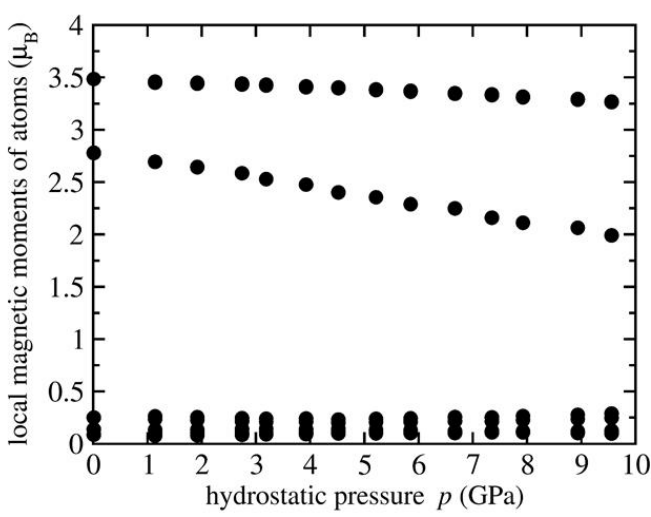

Figure 2 A visualization of 16-atom supercell of austenitic stoichiometric $\mathrm{Ni}_{2} \mathrm{MnSn}$ with two $\mathrm{Mn}-\mathrm{Ni}$ swaps (a). Part (b) includes the values of local magnetic moments of $\mathrm{Mn}$ and $\mathrm{Ni}$ atoms that are numerically listed in Bohr magnetons, indicated by the diameter of spheres representing the atoms and their orientation is shown by the arrows. The calculated pressure dependence of the total magnetic moment of the 16-atom supercell is shown sub-figure (c) and those of the local magnetic moments of constituent atoms are exhibited in subfigure (d). The local magnetic moments lower than $0.5 \mu_{\mathrm{B}}$ are those of $\mathrm{Ni}$ atoms while values above $1.9 \mu_{\mathrm{B}}$ represent those of $\mathrm{Mn}$ atoms. Magnetic moments of $\mathrm{Sn}$ atoms are lower than $0.1 \mu$ в and are not shown. 
This behavior is in contrast to that found in most magnetic systems where a lower volume typically causes a decrease in the magnetic moment (see, e.g. Refs. [7,11]). In order to shed a light on our findings, we have evaluated pressure-induced changes in the magnitudes of local magnetic moments and we present them in Figure 2(d). Importantly, all the local magnetic moments of $\mathrm{Mn}$ atoms behave as is typical for magnetic systems, i.e. the magnitude of their local magnetic moments decreases with increasing pressure (decreasing volume). But their pressure dependences differ. The swapped $\mathrm{Mn}$ atoms on the Ni sublattice are in different atomic environment than the $\mathrm{Mn}$ atoms on the Mn sublattice. As a consequence, not only that the magnitude of local magnetic moments of the swapped $\mathrm{Mn}$ atoms is lower and their orientation is antiparallel to that of the total magnetic moment, as discussed above, but also their pressure dependence is different. In particular, a slope of their decrease as a function of the pressure is steeper than the slope of $\mathrm{Mn}$ atoms on the Mn sublattice (that also have higher magnitude of their local magnetic moments, see Figure 2(d)). The swapped atoms are more sensitive to the applied hydrostatic pressures. The anomalous trend of the magnitude of the total magnetic moment is, therefore, partly caused by the fact that this magnitude is a sum of pressure-dependent local magnetic moments with some of them contributing with an opposite sign due to the ferromagnetic nature of this state. A major part of the total magnetic moment of the whole supercell is the difference between the magnitudes of two $\mathrm{Mn}$ atoms on the $\mathrm{Mn}$ sublattice with parallel orientation to that of the total magnetic moment (positive contributions) and the magnitudes of the two swapped $\mathrm{Mn}$ atoms on the Ni sublattice with antiparallel orientation to the total magnetic moment (negative contributions).

As far as pressure-dependences of local magnetic moments of $\mathrm{Ni}$ atoms are concerned, their magnitudes typically decrease or stay constant. Some of the $\mathrm{Ni}$ atoms have the magnitude of their local magnetic moments very slightly increased by the applied pressure but the increase is within an expected error bar of our calculations, e.g. an increase from $0.08 \mu_{\mathrm{B}}$ to $0.11 \mu \mathrm{B}$ in the case of the application of $9.6 \mathrm{GPa}$ of hydrostatic pressure, and do not represent any important physical phenomenon. It is worth noting that the atomic distribution of two Mn-Ni swaps per 16-atom supercell of the stoichiometric $\mathrm{Ni}_{2} \mathrm{MnSn}$ reduces the symmetry from a cubic one in the case of a defect-free Heusler structure (see Figure 1) to a tetragonal one. Therefore, there are two lattice parameters, see them defined in Figure 2(a), and each of them responds differently to the applied pressures. Consequently, their ratio changes with the pressure and the trend is provided in Figure 3(a). Interestingly, the zero-pressure state has the c/a ratio lower than one but increases with pressure, reaches the value of 1 for about $7.6 \mathrm{GPa}$, and is higher than one for yet higher pressures. For the sake of completeness, we also present the changes in the overall volume, see Figure 3(b). Last, but not least, it should be emphasized that the studied ferrimagnetic state (with the anomalous pressure-dependence of the total magnetic moment) is an excited state with respect to thermodynamically more stable ferromagnetic state that has the energy lower by about $18 \mathrm{meV} / \mathrm{atom}$.

(a)

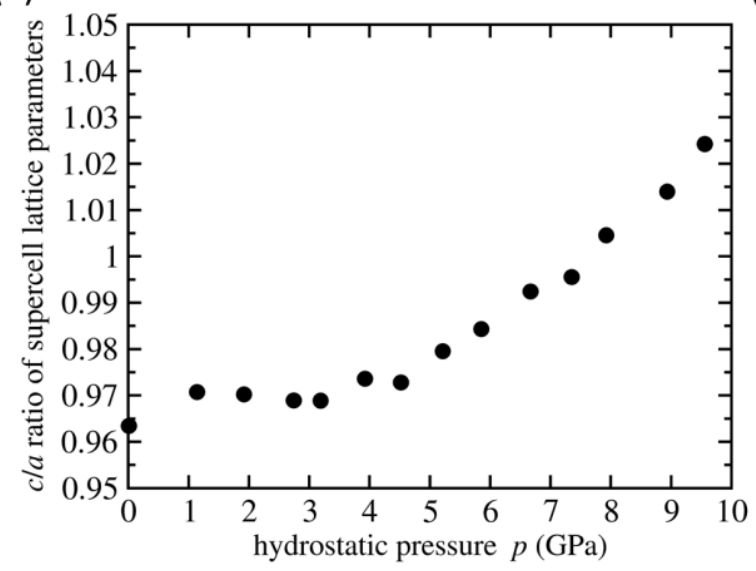

(b)

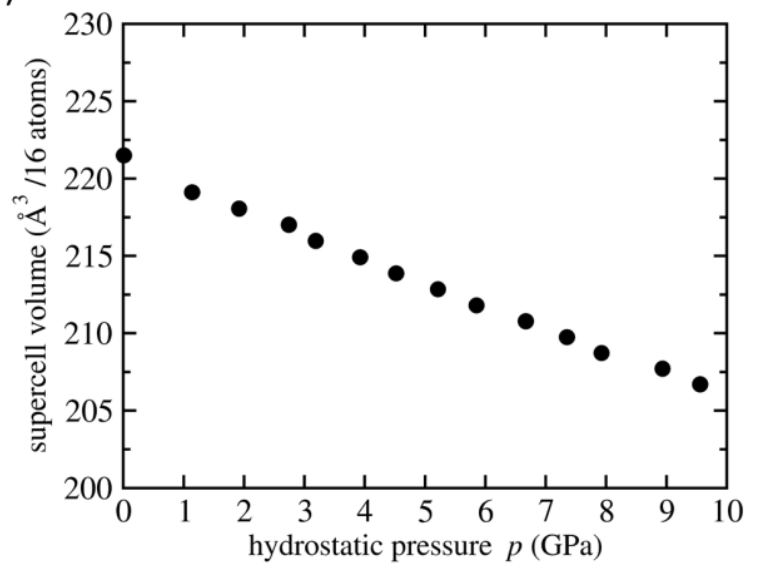

Figure 3 Computed pressure dependences of the ratio of lattice parameters $c / a(a)$ and the volume (b) of our computational 16-atom supercell of the stoichiometric $\mathrm{Ni}_{2} \mathrm{MnSn}$ with two Mn-Ni swaps. 
Next we present our results obtained for a highly off-stoichiometric $\mathrm{Ni}_{2} \mathrm{MnSn}$ state with the composition $\mathrm{Ni}_{7} \mathrm{Mn}_{8} \mathrm{Sn}_{1}$ that has a cubic symmetry, i.e. an austenite. The 16-atom supercell is schematically shown in Figure 4(a). There is one $\mathrm{Mn}$ atom on the Ni sublattice, the replaced $\mathrm{Ni}$ atom is completely missing and there are three extra $\mathrm{Mn}$ atoms replacing three $\mathrm{Sn}$ atoms within the 16-atom supercell. The zero-pressure ferrimagnetic state is shown in Figure 4(b). The Mn atom on the Ni sublattice has a lower magnitude of its magnetic moment and this magnetic moment is antiparallel with respect to the orientation of the total magnetic moment (similarly as discussed above for $\mathrm{Mn}$ atoms on the Ni sublattice in the case of $\mathrm{Ni}_{2} \mathrm{MnSn}$ with two Mn$\mathrm{Ni}$ swaps). Magnetic moments of all Mn atoms on the Sn sublattice are also antiparallel but their magnitudes are similar to those of $\mathrm{Mn}$ atoms on the $\mathrm{Mn}$ sublattice. The pressure dependence of the total magnetic moment is again anomalous but the trend is different, convex, see Figure 4(c), when compared with the concave trend in the case of $\mathrm{Ni}_{2} \mathrm{MnSn}$ with two Mn-Ni swaps, see Figure 2(c). The anomaly can be again tracked down to the behavior of magnetic moments of individual Mn atoms. The magnitudes of their magnetic moments decrease with increasing pressure, i.e. they do not exhibit any anomalous pressure dependence. But their responses to the applied pressures are differ for different $\mathrm{Mn}$ atoms. Also this ferrimagnetic state is an excited one with respect to a state that exhibits $\mathrm{Mn}$ atoms on the $\mathrm{Mn}$ and $\mathrm{Sn}$ sublattices having parallel orientation of their magnetic moments (the energy is then lower by $23 \mathrm{meV} / \mathrm{atom}$ ).

(a)

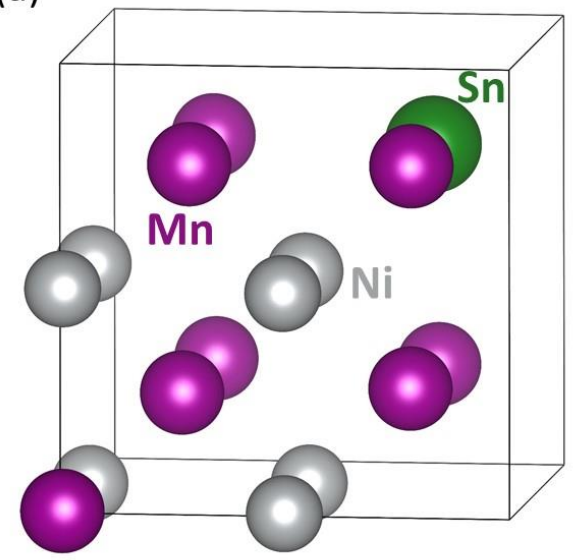

(c)

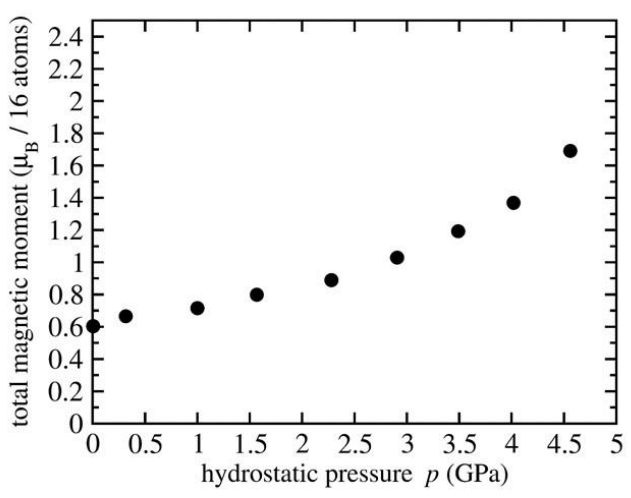

(b)

(d)
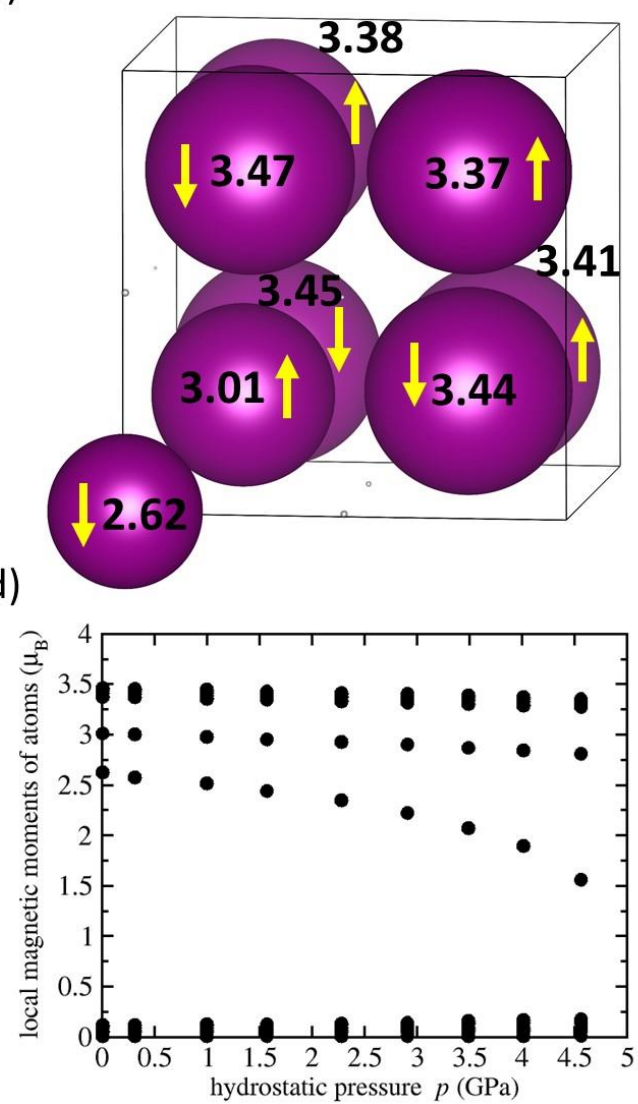

Figure 4 Schematic visualization of our 16-atom computational supercell of the studied austenite-structure off-stoichiometric Ni-Mn-Sn alloy with the composition $\mathrm{Ni}_{7} \mathrm{Mn}_{8} \mathrm{Sn}_{1}$ (a). Part (b) contains the magnitudes of local magnetic moments of $\mathrm{Mn}$ and $\mathrm{Ni}$ atoms (in Bohr magnetons) as indicated by the diameter of spheres representing the atoms and their orientation is shown by the arrows. The calculated pressure dependence of the total magnetic moment of the 16-atom supercell is shown sub-figure (c) and those of the local magnetic moments of constituent atoms are exhibited in sub-figure (d). The local magnetic moments lower than $0.5 \mu \mathrm{B}$ are those of $\mathrm{Ni}$ atoms while values above $1.5 \mu_{\mathrm{B}}$ represent those of $\mathrm{Mn}$ atoms. Magnetic moments of $\mathrm{Sn}$ atoms are lower than $0.1 \mu \mathrm{B}$ and are not shown. 


\section{CONCLUSION}

We have performed an ab initio study of two ferrimagnetic states, a Ni2 MnSn state with Mn-Ni swaps and an off-stoichiometric $\mathrm{Ni}_{7} \mathrm{Mn}_{8} \mathrm{Sn}_{1}$ alloy, modelled by 16 -atom supercells with the atomic positions related to those in the full Heusler structure. We have determined thermodynamic, magnetic and structural properties of the both pressure-free states as well as those corresponding to hydrostatic pressures of a few GPa. Both studied states exhibit anomalous pressure-induced changes in the total magnetic moment when the total magnetic moments of the supercells increase with increasing hydrostatic pressure. Despite of this peculiar trend in the total magnetic moment, the magnitudes of local magnetic moments of individual $\mathrm{Mn}$ atoms (that play a decisive role) decrease with increasing hydrostatic pressure. The identified anomalous phenomena may be related to an interplay of a few atomic-level mechanisms. First, the magnetic moments of $\mathrm{Mn}$ atoms, that are either parallel or antiparallel to the orientation of the total magnetic moment of the supercells, nearly compensate each other due to the ferrimagnetic nature of the studied magnetic states. Second, the swapped and offstoichiometric atoms lead to different local atomic environments of $\mathrm{Mn}$ atoms and, consequently, to different local magnetic moments of these $\mathrm{Mn}$ atoms as well as their different response to hydrostatic pressures. Importantly, the local magnetic moments of $\mathrm{Mn}$ atoms, that are antiparallel to the orientation of the total magnetic moment, are more sensitive to the applied pressures. Regarding thermodynamic properties, the studied states are excited ones. As the next step we plan to test the mechanical stability of the studied states by evaluating their elastic constants and phonon spectra similarly as done in Ref. [19]

\section{ACKNOWLEDGEMENT}

The authors acknowledge the Czech Science Foundation for the financial support received under the Project No. 20-16130S. Computational resources were provided by the Ministry of Education, Youth and Sports of the Czech Republic under the Projects e-INFRA CZ (ID:90140) at the IT4Innovations National Supercomputing Center and e-Infrastructure CZ (e-INFRA LM2018140) at the MetaCentrum as well as CERIT Scientific Cloud, all provided within the program Projects of Large Research, Development and Innovations Infrastructures. Sub-figures $2(a, b)$ and $4(a, b)$ were visualized using the VESTA software [20].

\section{REFERENCES}

[1] HEUSLER, F.; STARCK, W.; HAUPT, E. Über die Synthese ferromagnetischer Manganlegierungen. Verhandlungen der Deutschen Physikalischen Gesellschaft. 1903, vol. 5, p. 219.

[2] GRAF, T.; FELSER, C.; PARKIN, S.S.P. Simple rules for the understanding of Heusler compounds. Progress in Solid State Chemistry. 2011, vol. 39, p. 1.

[3] RECARTE, V.; et al. Direct evidence of the magnetoelastic interaction in $\mathrm{Ni}_{2} \mathrm{MnGa}$ magnetic shape memory system. Applied Physics Letters. 2013, vol. 102, Art. No. 201906.

[4] LEE, S. J.; et al. Magnetic, magneto-optical, and transport properties of ferromagnetic shape-memory $\mathrm{Ni}_{2} \mathrm{MnGa}$ alloy. Journal of Applied Physics. 2003, vol. 93, pp. 6975-6977.

[5] BROWN, P. J.; et al. The crystal structure and phase transitions of the magnetic shape memory compound $\mathrm{Ni}_{2} \mathrm{MnGa}$. Journal of Physics: Condensed Matter. 2002, vol. 14, pp. 10159-10171.

[6] ENTEL, P.; et al. Complex magnetic ordering as a driving mechanism of multifunctional properties of Heusler alloys from first principles. The European Physics Journal B. 2013, vol. 86, no. 65.

[7] KAŠTIL, J.; et al. Effect of pressure and high magnetic field on phase transitions and magnetic properties of $\mathrm{Ni}_{1.92} \mathrm{Mn}_{1.56} \mathrm{Sn}_{0.52}$ and $\mathrm{Ni}_{2} \mathrm{MnSn}$ Heusler compounds. Journal of Alloys and Compounds. 2015, vol. 650, pp. 248255.

[8] SUTOU, Y.; et al. Magnetic and martensitic transformations of $\mathrm{NiMnX}(\mathrm{X}=\mathrm{In}, \mathrm{Sn}, \mathrm{Sb})$ ferromagnetic shape memory alloys. Applied Physics Letters. 2004, vol. 85, pp. 4358-4360.

[9] BROWN, P.J.; et al. The magnetic and structural properties of the magnetic shape memory compound $\mathrm{Ni}_{2} \mathrm{Mn}_{1.44} \mathrm{Sn}_{0.56 .}$ Journal of Physics: Condensed Matter. 2006, vol. 18, pp. 2249-2259.

[10] ÇAKIR, A.; et al. Intermartensitic transitions and phase stability in $\mathrm{Ni}_{50} \mathrm{Mn}_{50-x} \mathrm{Sn}_{\mathrm{x}}$ Heusler alloys. Acta Materialia. 2015, vol. 99, pp. 140-149. 
[11] FRIÁK, M.; et al. An ab initio study of pressure-induced changes of magnetism in austenitic stoichiometric $\mathrm{Ni}_{2} \mathrm{MnSn}$. Materials. 2021, vol. 14, no. 523.

[12] KRESSE, G., HAFNER, J. Ab initio molecular dynamics for liquid metals. Physical Review B. 1996, vol. 47, pp. 558-561.

[13] KRESSE, G., FURTHMÜLLER, J. Efficient iterative schemes for ab initio total-energy calculations using a planewave basis set. Physical Review B. 1996, vol. 54, pp. 11169-11186.

[14] HOHENBERG, P., KOHN, W. Inhomogeneous electron gas. Physical Review B. 1964, vol. 136, pp. B864-B871.

[15] KOHN, W., SHAM, L. J. Self-consistent equations including exchange and correlation effects. Physical Review $A$. 1965, vol. 140, pp. A1133-A1138.

[16] BLÖCHL, P. E. Projector augmented-wave method. Physical Review B. 1994, vol. 50, pp. 17953-17979.

[17] KRESSE, G., JOUBERT, D. From ultrasoft pseudopotentials to the projector augmented-wave method. Physical Review B. 1999, vol. 59, pp. 1758-1775.

[18] PERDEW, J. P., BURKE, K., ERNZERHOF, M. Generalized gradient approximation made simple. Physical Review Letters. 1996, vol. 77, pp. 3865-3868.

[19] FRIÁK, M., et al. Pressure-induced increase of the total magnetic moment in ferrimagnetic $\mathrm{Ni}_{1.9375} \mathrm{Mn}_{1.5625} \mathrm{Sn}_{0.5}$ martensite: A quantum-mechanical study. accepted for publication in Materials Transactions (2022), Available from: https://doi.org/10.2320/matertrans.MT-MA2022006.

[20] MOMMA, K., IZUMI, F. VESTA 3 for three-dimensional visualization of crystal, volumetric and morphology data. Journal of Applied Crystalography. 2011, vol. 44, pp. 1272-1276. 